\title{
Correction to: A meta-analysis of the relationship between vaginal microecology, human papillomavirus infection and cervical intraepithelial neoplasia
}

Yuejuan Liang, Mengjie Chen, Lu Qin, Bing Wan and He Wang*

Correction to: Infect Agents Cancer (2019) 14: 29

https://doi.org/10.1186/s13027-019-0243-8

In the original publication of this article [1] there was an error in the results section of the article.

The result section showed the following:

- The total results based on all eight studies (OR 2.62, 95\% CI 1.84-3.73, P < 0.05) were statistically significant (Fig. 1)

However, the correct information is:

- The total results based on all eight studies (OR 2.57, 95\% CI 1.78-3.71, P < 0.05) were statistically significant (Fig. 1)

The updated information is shown in bold. The correct information is already available in Fig. 1 of the original publication.

Published online: 09 December 2019

\section{Reference}

1. Liang $Y$, Chen $M$, Qin $L$, et al. A meta-analysis of the relationship between vaginal microecology, human papillomavirus infection and cervical intraepithelial neoplasia. Infect Agents Cancer. 2019;14:29. https://doi.org/10. 1186/s13027-019-0243-8. 